\title{
Proboitics and their Role in GI Diseases
}

\author{
S PERVEEN ${ }^{\mathrm{a}}$, MAAHMED ${ }^{\mathrm{b}}$
}

\begin{abstract}
Summary:
Objective: To evaluate role of probiotics in human physiology, metabolism, health, immunity and GI disorders.It is important for gastroenterologists to improve their understanding of the mechanisms of probiotics and the evidence that support their use in clinical practice.
\end{abstract}

Data Sources: A medline search (1948-December 2014) was conducted using GI diseases and probiotics as terms for identifying pertinent studies. Search limits included English language. Additional information was obtained from bibliographies.

Data Selection And Data Extraction: The information provided is based on review of primary literature from randomized controlled trials (RCTs), meta-analyses, expert consensus panel recommendations and society-based practice recommendations. References are provided for more reading and figure summarizes key information about their mechanism of action.

Data Synthesis: The need for objective, evidence-based guidance on the role of probiotics is becoming increasingly

Introduction:

Our gut is home to an estimated 100 trillion microorganisms representing more than1000 different bacterial species, 400 of which are estimated to be of probiotic species ${ }^{1}$. There is growing recognition of the role of diet and other environmental factors in modulating the composition and metabolic activity of the human gut microbiota, which in turn can impact health.Up to $10 \%$ of an individual's daily energy needs can be derived from the by products of bacterial fermentation. Gastrointestinal microbiota are also critical for normal immune system development. ${ }^{2}$ Intestinal

a. Colonel (Dr) Shaila Perveen, Classified Medical Specialist and Gastroenterologist, CMH Jessore.

b. Dr. Colonel Mir Azimuddin Ahmed, Classified Medical Specialist and Gastroenterologist, CMH Jessore

Address of Correspondence: Colonel (Dr) Shaila Perveen, Classified Medical Specialist and Gastroenterologist, CMH Jessore.

Received: 22 March, 2015

Accepted: 30 March, 2016 important as public awareness grows. This consensus is intended as a practical reference to help physicians make appropriate, evidence-based recommendations to patients who might benefit from probiotic treatment. Overall, the randomised, placebo-controlled trials included in this article support, with a high evidence level, the therapeutic effects of probiotic agents for several disorders including antibiotic or Clostridium difficile-associated diarrhea, irritable bowel syndrome, and the inflammatory bowel diseases. Although probiotic research is a rapidly evolving field, there are sufficient data to justify a trial of probiotics for treatment or prevention of some of these conditions. However, the capacity of probiotics to modify disease symptoms is likely to be modest and varies among probiotic strains and not all probiotics are right for all diseases. The goal of this review is to provide clinicians with an overview of the rationale and data which support or refute the role of probiotics for treating commonly encountered gastrointestinal disorders.

(J Bangladesh Coll Phys Surg 2016; 34: 92-99)

bacteria weigh up to $1 \mathrm{~kg}$ and bacterial cells outnumber human cells by 10:1. The bacterial genome or DNA may outnumber the human genome by 100:1.The physiologic impact mediated by these resident microbes is important enough to be labeled as "other organ" or " hidden metabolic organ"

Probioticsare defined as 'live microorganisms' which when administered in adequate amounts confer a health benefit on the host'. ${ }^{3}$ There is plentiful evidence that probiotics( pro and biota meaning 'for life' ) impact the microbiome and benefit human health ${ }^{4}$. Probiotics are distinct from prebiotics (dietary substances such as indigestible oligosaccharides including bananas, whole grains, honey, garlic and onions) that provide a health benefit by selectively promoting the growth of beneficial bacteria in the gut and synbiotics (products containing a synergistic combination of prebiotics and probiotics ).

Although research on the microbiome is emerging, scientists have already made tremendous progress in 
understanding the microbial makeupand associating microbiome diversity with human physiology,health and disease. It is likely that much of this impact is mediated through diet. What we eat and drink influences the microbiome and the microbiome in turn converts dietary content into biological signals providing enzymatic machinery thus influencing what the human host is able to extract from its diet including energy. ${ }^{5}$

\section{Probiotics and the Immune System}

Humans and their trillions of intestinal microorganisms coevolve to form a largely beneficial symbiosis. Human gut microbiome is acquired during the last trimester of pregnancy $^{6}$. Unlike the human genome, the human microbiome is acquired. Vaginally born babies acquire different microbiomes than babies born by cesarean section $^{7}$. Gastrointestinal microbiota are critical for normal immune system development ${ }^{8}$. The intestinal mucosa comprising the largest surface area of the body, is constantly exposed to a vast array of microbes, food antigens, and toxins. The intestinal epithelium must 'tolerate' the commensal flora that maintain mucosal homeostasis by controlling inflammatory responses as well as sensing danger signals of potentially harmful pathogens.

Probiotics can down regulate the effects of luminal bacteria in initiating and sustaining an intestinal inflammatory response particularly important in ulcerative colitis. It is becoming increasingly clear that the intestinal microbiota composition, the intestinal barrier and the mucosal immune system plays pivotal roles in the development of a variety of allergic and autoimmune diseases.

\section{Types of Probiotics}

Most of the probiotics are bacteria, some are fungus(yeast) and some contain mixture.Lactobacillus acidophilus is the "friendly" bacteria and most commonly used probiotic. Such healthy bacteria inhabit the intestines and protect against the entrance and proliferation of pathogens. Since the mid 1990s, clinical studies have established that probiotic therapy can help treat several gastrointestinal illnesses. ${ }^{9}$

Lactobacillus acidophilus, Lactobacillus casei both convert lactose into lactic acid helping in lactose intolerance. Lactobacillus bulgaricus(discovered by Bulgarian doctor) can be found inmany yogurts and soft cheese.Itis helpful for those who are lactose intolerant $L$. Acidophilus may also be helpful in reducing cholesterol levels.Lactobacillus rhamnosus GG means the genus is Lactobacillus, the species is rhamnosus and the strain is GG. ${ }^{10}$

Bifidobacteria is a family of bacteria that has been studied for its ability to prevent and treat various gastrointestinal disorders, including infections, irritable bowel syndrome and constipation. In addition to making lactic acid, it also makes some important short-chain fatty acids that are then absorbed and metabolized by the body. Certain bifidobacteria may actually protect the host from carcinogenic activity of other intestinal flora.

Saccharomyces boulardii is the only yeast probiotic. Some studies have shown that it is effective in preventing and treating diarrhea associated with the use of antibiotics and to reduce side effects of $H$. pylori therapy, traveler's diarrhea,Crohn's disease.

Probiotic mixture (VSL\#3 )consisting of 8 strains of live freeze-dried lactic acid bacteria. Each sachet contains 450 billion live probiotic bacteria .Streptococcus thermophilus, Bifidobacterium breve, B longum, B infanti, Lactobacillus acidophilus, L plantarum, L paracasei, L bulgaricus. ${ }^{11}$

Probiotic therapy may also help people with Crohn's disease and irritable bowel syndrome. Clinical trial results are mixed, but several small studies suggest that certain probiotics may help maintain remission of ulcerative colitis and prevent relapse of Crohn's disease and the recurrence of pouchitis (a complication after surgery for ulcerative colitis). Because these disorders are so frustrating to treat, many people are giving probiotics a try even before all the evidence is in for the particular strains. More research is needed to find out which strains work best for what conditions.

\section{Mechanism of Action of Probiotics}

Probiotics work by several different mechanisms. These commensal microorganisms contribute energy and cellular precursors in the form of short-chain fatty acids, prevent infections and modulate and train the host immune system. ${ }^{12}$ They act as a barrier by lining the intestinal tract close to the brush border. Through competitive inhibition, they prevent other luminal bacteria stimulating the mucosal immune system. They enhance mucus production so that patients will have a thicker mucus layer, which protects against invasive bacteria. Probiotics influence the mucosal immune system to secrete protective immunoglobulins (Ig) such as secretory IgA and protective defensins and bacteriocins into the lumen (Fig I). Finally, probiotics alter the function of the mucosal immune system to make 


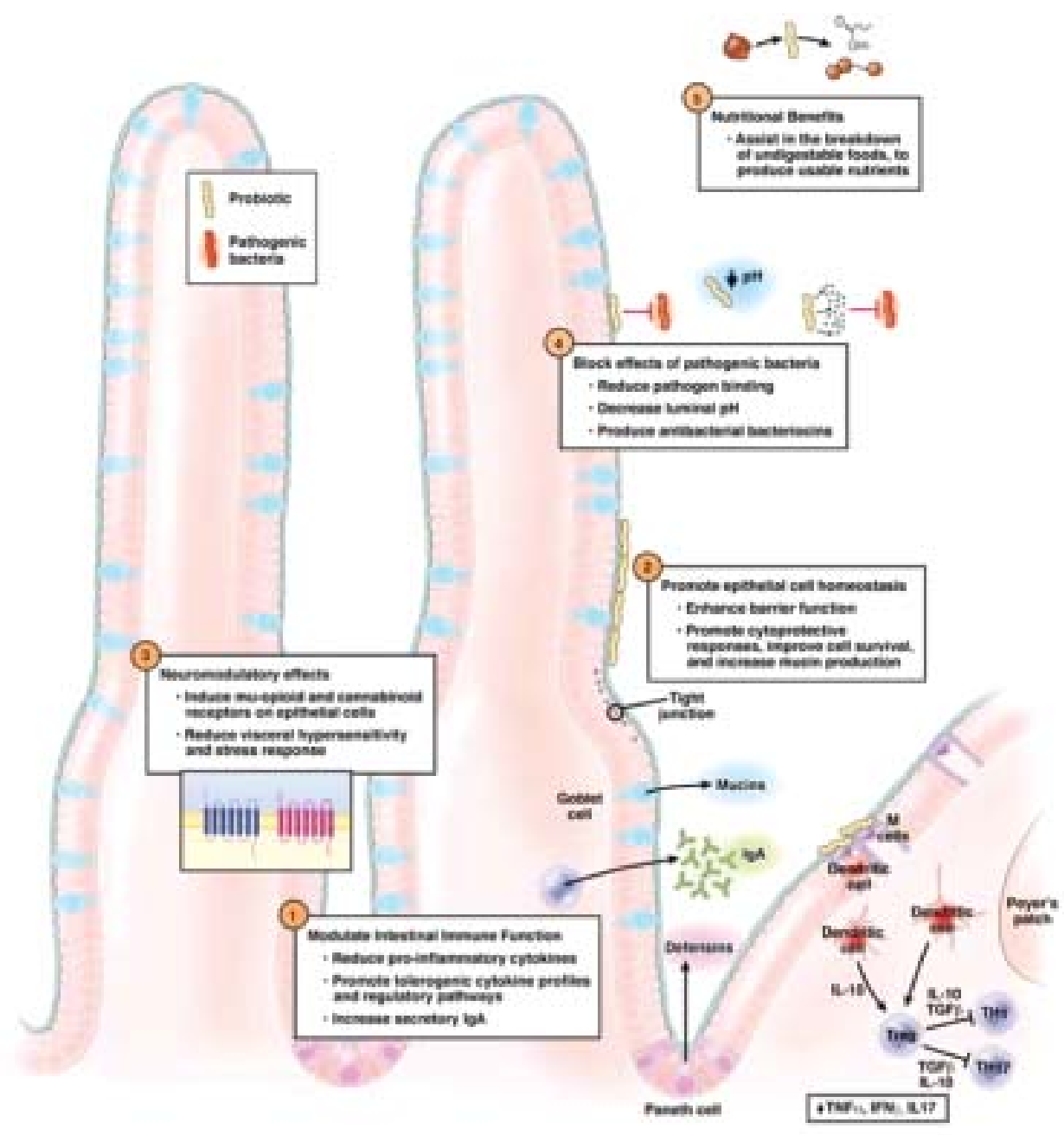

Fig.-1: Diagrammatic representation of mechanism of action of Proboitics

dendritic cells more anti-inflammatory and less proinflammatory so that they are slightly less responsive and less reactive to luminal bacteria in initiating and sustaining an intestinal inflammatory response which is particularly important in ulcerative colitis. ${ }^{13}$

\section{Indications of Probiotics}

Altough still being studied, probiotics may help several specific illnesses. In 2011, experts at Yale University reviewed the research studies. They concluded that probiotics are best case in the treatment of diarrhea. Controlled trials have shown that Lactobacillus GG can shorten the course of infectious diarrhea in infants and children (but not adults). Although studies are limited and data are inconsistent, two large reviews, taken together, suggest that probiotics reduce antibioticassociated diarrhea by $60 \%$ when compared with a placebo. Probiotics can improve intestinal function and maintain the integrity of the intestinallining and help fight bacteria that cause diarrhea. In childhood diarrheain the pediatric population, rotavirus has been the most common cause of infectious diarrhea. Data suggests that the benefit of probiotics in preventing acute infectious diarrhea is modest. ${ }^{14}$

In treating necrotizing enterocolitis, a type of infection and inflammation mostly seen in infants Lactobacillus rhamnosus GG (LGG) is the most effective probiotic reported to date, reducing both severity and duration of diarrhea by $\sim 1$ day. ${ }^{15}$ The American Academy of Pediatrics supports the recommendation of LGG early in the course of acute infectious diarrhea to reduce symptom duration. 
For antibiotic associated diarrhea incuding those receiving Helicobacter pylori eradication therapy and viral gastroenteritis supporting data are strong for probiotics (L. rhamnosusGG, Saccharomyces boulardii)are among the few treatment modalities available. However, the duration of symptoms in these conditions is typically short regardless of probiotic use. Patients given the higher dose of probiotic concurrent with antibiotics ( for 5 days afterward) had fewer occurrences of AAD (15.5 vs. 44.1\%).As a secondary endpoint these studies also showed a reduction in development of C. difficile-associated diarrhea ${ }^{16}$.

Diarrhea caused by $C$. difficile bacteria is a common nosocomial and community-based medical condition. $C$ difficile sickens half a million Americans and kills more than approximately 14,000 people a year in the United States. ${ }^{17}$ Antibiotic therapy with metronidazole, oral vancomycin and now fidaxomicin makeup the current treatment regimen but recurrence remains a clinical problem.

Recently, In patients with recurrent CDAD the fecal communities were highly variable in bacterial composition and were characterized by markedly decreased diversity. Preservation and restoration of the microbial diversity could represent novel strategies for prevention and treatment of recurrent CDAD. ${ }^{18}$

Fecal microbiota transplants (FMTs), also known as stool transplants from healthy donors have emerged as an effective means of stopping infections and succeeded in $91 \%$ of patients with recurrent CDI.Patients who underwent colonoscopic FMT found it so effective that more than half $(53 \%)$ said that a fecal microbiota transplant would be their top choice if they contracted CDI again. 19,20

Typically, patients receive the fecal transplants by enema, colonoscopy or a nasal tube.As a potentially less costly and less invasive alternative, researchers made fecal pill.Dr. Louie first created a fecal transplant pill when a $C$ difficile infection persisted in one patient who could not tolerate a nasal tube. ${ }^{[21]}$ Currently, it is approved only for recurrent $C$ difficile infections,

After the patients ingested the capsules, the researchers found significantly increased numbers of Bacteroides, C coccoides, C leptum, Prevotella, Bifidobacteria, andDesulfovibrio and significantly decreased numbers ofEnterobacteriaceaeandVeillonella. They found no $C$ difficile in the intestines.
Symptoms of irritable bowel syndrome (IBS) and other functional GI disorders (FGID) frustrate people with overlapping symptoms and exhaust them with prolonged conventional medications having considerable adverse effects. ${ }^{22}$ They can be treated with foods and supplements containing probiotics.

The first practical consensus on the role of probiotics in the management of GI symptoms in adultsrecommend specific probiotics in the management of some IBS symptoms and can also be used as an adjunct to conventional treatment. ${ }^{23}$ For overall symptoms and abdominal pain in IBS, probiotics(Bifidobacterium bifidumMIMBb75,Escherichia coliDSM17252) has strong supportive evidence for benefit and should be tried.In overall symptoms in IBS-D(B. longum subsp. infantis 35624), improving the frequency and/or consistency of bowel movements and bloating/ distension (B. animalis subsp. lactis DN-173 010 Activia) probiotics could be tried.In overall symptoms in IBS-C(B. animalis -Activia) probiotics may be considered.Flatus in IBS has currently no evidence to support use of probiotics. No probiotic alleviates the full range of symptoms in IBS. ${ }^{24}$ Though it has shown improvement in some aspects of health-related quality of lifethere is need for more research.This consensus publication with new research and input from patient groups is intended to be updated in 3 years.

The need for objective, evidence-based guidance on the role of probiotics is becoming increasingly important as public awareness for probiotics grows. This consensus is intended as a practical reference to help physicians make appropriate, evidence-based recommendations to patients who might benefit from probiotic treatment. Overall, the randomised, placebocontrolled trials included with a high evidence level, a role for specific probiotics in the management of overall symptoms and abdominal pain in patients with IBS. Preventing or reducing diarrhoea in patients receiving antibiotics or $H$. pylori eradication triple therapy. The trials support, with a moderate evidence level, a role for specific probiotics in managing overall symptoms in patients with IBS-D; improving bowel movements and bloating/distension in patients with IBS; and improving some aspects of health-related quality of life. ${ }^{25}$

The lack of consensus on the role of probiotics in the management of constipation is consistent with the World Gastroenterology Organisation guideline on 
prebiotics and probiotics, which recommends certain prebiotics but not probiotics, for the treatment of constipation. $^{26}$

Inflammatory bowel disease (IBD) e g Crohn's disease, ulcerative colitis and pouchitis(after ileal pouch anal anastomosis in ulcerative colitis patients) are often refractory to standard therapy. The rational to use probiotics and its beneficial efficacy in the treatment of chronic inflammatory bowel disease (IBD) is increasingly scrutinized.The role of probiotics for inducing and maintaining remission especially regarding induction of remission for ulcerative colitis has shown breakthroughs. ${ }^{27}$

A relationship between immune response and gastrointestinal microbials appears to be involved in the mechanism of ulcerative colitis (UC). Alternative IBD treatment approaches aimed at modifying the composition of the intestinal microbiota in order to overcome gut dysbiosis have become a subjectof major interest in recent years. There is also some evidence that probiotics may facilitate and stabilize clinical remission. ${ }^{28}$

Changes in the relative abundance of the families Enterobacteriaceae and Lachnospiraceae in UC patients provide useful diagnostic indications of clinical response after FMT. ${ }^{29}$ Randomized, placebo-controlled trials with larger cohorts will be necessary to establish cause-effect relationships for the successfully transmitted donor phylotypes such as F. prausnitzii, B. ovatus, and $R$. faecis.

Trial results comparing the probiotic(Escherichia coli Nissle1917) tomesalazine have reported equivalent rates of UC relapse. Treatment with Lactobacillus rhamnosus GGstrain alone or in combination with mesalazine resulted in a nonsignificant odds ratio decrease for relapse and a significant increase in time to relapse compared to treatment with mesalazine alone. Additionally, bifidobacteria fermented milk supplemented patients had significant reductions in UC exacerbations when compared to nonsupplemented patients. Probiotics were well tolerated, with adverse event rates similar between treatments. One study of 90 volunteers found significantly higher remission rates in people with ulcerative colitis who were given the beneficial bacteria E. coli Nissle. The higher the dose, the longer their remission. ${ }^{30}$
It is suggested that patients with ulcerative colitis benefit by using bacterial therapies and patients with Crohn's disease from S. boulardii, an yeast. ${ }^{31}$

Chronic or recurrent pouchitis is an important complication occurring in $~ 10-20 \%$ of UC patients after ileal anal pouch formation surgery. VSL\#3 was shown beneficial in prophylaxis against pouchitis onset after surgical take-down and in maintaining clinical remission after antibiotic induction.Clinical expert guidelines concur that probiotics (VSL\#3) can be effective for preventing recurrence of pouchitis. ${ }^{32,33}$

Diverticulosis is present in approximately two thirds of the elderly population and a large majority of those affected remain entirely asymptomatic. However, an estimated $20 \%$ of patients may develop 'diverticular disease' with time. Change in the colonic microflora, resulting in a decrease in healthy flora and an increase in pathogenic bacteria, may be detected in patients with diverticular disease. This may allow chronic inflammation and epithelial cell proliferation in and around the diverticula. ${ }^{34}$ Probiotics, restoring the colonic microenvironment, have been proposed to treat those patients. ${ }^{35}$

The most recently published RCT in Diverticulosis compared mesalazine or mesalazine plus Bifidobacterium infantis 35624 for 12 weeks and followed up for nine additional months. Global symptom scores were assessed over the 52 weeks of followup.Mesalamine demonstrated a consistent trend in reducing symptoms. Addition of probiotic did not increase mesalamine efficacy. ${ }^{36}$

Another Multicentre Double-blind, Randomised, Placebo-Controlled Study showed both cyclic mesalazine and $L$. casei subsp. $D G 24$ billion/day appear to be better than placebo for maintaining remission in symptomatic uncomplicated diverticular disease (SUDD) especially when used in combination. Moreover, both treatments alone or in combination are significantly better than placebo in preventing occurrence of acute diverticulitis in SUDD patients. ${ }^{37}$

Alcoholic liver disease is characterized by fatty liver (steatosis), which may progress to alcoholic hepatitis, fibrosis, and cirrhosis. ${ }^{38}$ Intestinal bacterial overgrowth is common in patients with alcoholic liver disease. In alcoholics, translocation of bacteria and bacterial products into the circulation contributes to liver disease. 
Treatment with prebiotics partially restored gut microbiome, reduced bacterial overgrowth and lessened alcoholic steatohepatitis. Disbiosis (disruption or dysregulationof intestinal antimicrobial molecules) contributed to changes in the enteric microbiome leading to alcoholic steatohepatitis in mice ${ }^{39}$

Role in Metbolic disease or obesity shows comparisons of the distal gut microbiota of genetically obese and lean human volunteers revealing that obesity is associated with changes in the relative abundance of the two dominant bacterial divisions, the Bacteroidetesand the Firmicutes. Obese microbiome has an increased capacity to harvest energy from the diet. ${ }^{40}$ Gut microbiota is an additional contributing factor to the pathophysiology of obesity. ${ }^{41}$ Studies in mice have associated the phylum Firmicutes with obesity and the phylum Bacteroidetes with weight loss. ${ }^{42}$

There is clear evidence that the intestinal microbiota influences the host through its effect on body weight, bile acid metabolism, proinflammatory activity and insulin resistance, and modulation of gut hormones ${ }^{43}$. A synergism between role ofmicroflora, aberrant intestinal microbiota(either quantitatively or qualitatively), a "leaky" gut mucosal barrier and altered mucosal immunity contributing to type 1 diabetes has begun to evolve. Changes in gut microbiota and thus cell wall components are involved in the epigenetic regulation of inflammatory reactions. An improved diet targeted to induce gut microbial balance and epigenetic changes of pro-inflammatory genes may be effective in the prevention of metabolic syndrome. ${ }^{44}$

\section{Precautions about Probiotics}

Probiotics preparations are generally considered as food not drug. They are already present in a normal digestive system and therefore safeand may cause few side effects.In the USA, formulations of probiotics are medical foods and must be used under medical supervision.In a systematic review of studies, rate of side effects was same in probiotics users as in those taking mesalazine.No serious side effects have been associated with prolonged use of probiotics in ulcerative colitis. ${ }^{45}$

Probiotics have enjoyed an impeccable safety reputation but immune compromise (including a debilitated state or malignancy) has been identified as a risk factor for rare cases of bacteraemia or fungaemia in patients taking certain probiotics (most commonly Saccharomyces boulardii). ${ }^{46}$ No serious adverse events attributed to FMT were observed. ${ }^{47}$

One high profile multicenter placebo controlled Dutch RCT examining probiotic supplementation in severe acute pancreatitis found a higher incidence of mesenteric ischemia and death in the treatment group. ${ }^{48}$ This is the only trial supporting the concept that probiotics should be avoided in critically ill patients.

Probiotic ingredients should be clearly marked on the label. There's no way to judge the safety of unidentified mixtures.Probiotics are food particles andthey are rapidly washed awaywithin days, although strainspecific differences occur. ${ }^{49}$ For a chronic GI problem, it is critically important that the product is taken in adequate doses on a regular basis (e.g. just before a meal) for a reasonable period of time, which should be at least a month, unless it cannot be tolerated for any reason. In order to get the full benefits of probiotics, dose selection should be based on available evidence and manufacturers' recommendations. Some yogurts contain the friendly bacteria but as they are sensitive to oxygen, light and dramatic temperature changesso when heat-treated or pasteurized, they lose thesevaluable"live and active cultures.".

Despite their long history, wide availability and substantial publication record, the clinical role of probiotics are in general, inadequately characterised and remains ill-defined. Probiotic research are complicated by the wide variety of probiotic strains obtained with one strain notapplicable to others. According to age and health status of the target group effects of probiotics may vary.

The variable range of formulations (capsules, sachets, yoghurts and fermented milks or fruit drinks), differ indose and the presence of supporting substrates add further sources of variation. ${ }^{50}$

\section{Conclusion:}

Despite widespread use of natural therapies by patients allured by advertisesments, health care providers may be unfamiliar with probiotics as a treatment modality.Many patients consume probiotic products in attempts to manipulate the intestinal microbiota for health benefit. Questions regarding optimal probiotic, dosing, specific patient populations and placement in therapy are to be answered by large, randomized, 
controlled trials conducted before probiotics can be routinely recommended.Evidence supports a role for considering the recommendation of conventional probiotics for some clinical conditions. Probiotic strain selection should focus on quality tested products with clinically demonstrated benefit for the given disorder. Patients and physicians should expect modest effects and consider using probiotics as a supplement rather than a replacement for conventional therapy. ${ }^{[51]}$

Probiotic research is evolving rapidly and this article reflect physicians' rather than patients' perspectives.Future clinicians will have the opportunity to use directed selection of a probiotic or probiotic derived product to specifically address a unique disease causing physiologic or genetic defect.Though challenges exist, ongoing investigations offer great promise for the future.

Some patients have interest in probiotics and their potential and they may take probiotics (or products incorrectly identified as probiotics) to reduce their symptoms before consulting their physician. Therefore, educational materials for the general public are also needed to improve their understanding and to ensure appropriate use of probiotics.

\section{References:}

1. Macpherson AJ, Harris NL, Interactions between commensal intestinal bacteria and the immune system. Nat Rev Immunol. 2004; 4(6):478-85.

2. Conlon MA, Bird AR. The Impact of Diet and Lifestyle on Gut Microbiota and Human Health. Nutrients. 2014;7(1):17-44.

3. FAO/WHO Working Group. Guidelines for the evaluation of probiotics in food. Report of a joint FAO/WHO working group on drafting guidelines for the evaluation of probiotics in food. London, Ontario, Canada 2002. Available at: ftp://ftp.fao.org/es/esn/food/wgreport2.pdf. Accessed 2013.

4. Sanders ME. Impact of probiotics on colonizing microbiota of the gut. Journal of Clinical Gastroenterology. 2011;45:115-119.

5. Thomas CM, Versalovic J. Probiotics-host communication: Modulation of signaling pathways in the intestine. Gut Microbes. 2010;1(3):148-163.

6. Koenig JE, Spor A, Scalfone N, Fricker AD, Stombaugh J, Knight R, Angenent LT, Ley RE. Succession of microbial consortia in the developing infant gut microbiome. PNAS. 2011;108(Suppl 1):4578-4585.
7. Dominguez-Bello MG, Costello EK, Contreras M, Magris M, Hidalgo G, Fierer N, Knight R. Delivery mode shapes the acquisition and structure of the initial microbiota across multiple body habitats in newborns. PNAS. 2010;107(26):11971-11975.

8. Macpherson AJ, Harris NL. Interactions between commensal intestinal bacteria and the immune system. Nat Rev Immunol. 2004; 4(6):478-85.

9. $\mathrm{R}$ Fuller. Probiotics in human medicine. Gut 1991;32(4):439-442.

10. H.M. Timmerman, C.J.M. Koningb, L. Mulderc, F.M. Romboutsd, A.C. BeynenaMonostrain. multistrain and multispecies probiotics-A comparison of functionality and efficacy.International Journal of Food Microbiology. 2004; 96(3):219-233.

11. Elizabeth C. Verna. Use of probiotics in gastrointestinal disorders: what to recommend? TherapAdvGastroenterol. 2010; 3(5): 307-319.

12. Borchers AT, Selmi C, Meyers FJ, Keen CL, Gershwin ME. Probiotics and immunity. Gut 2010; 59(3):325-32.

13. Richard N. Fedorak. Probiotics in the Management of Ulcerative Colitis. GastroenterolHepatol (N Y). 2010; 6(11): 688-690.

14. Thomas DW, Greer FR, American Academy of Pediatrics Committee on Nutrition, Review Probiotics and prebiotics in pediatrics. Pediatrics. 2010; 126(6):1217-31

15. Allen SJ, Martinez EG, Gregorio GV, Dans LF, Review Probiotics for treating acute infectious diarrhoea. Cochrane Database Syst Rev. 2010;10 (11):CD003048

16. Issa I, MoucariR.Probiotics for antibiotic-associated diarrhea: Do we have a verdict? World J Gastroenterol.2014;20(47):17788-17795.

17. Floch MH, Walker WA, Madsen K, Sanders ME, Macfarlane GT, Flint HJ, Dieleman LA, Ringel Y, Guandalini S, Kelly CP, Brandt LJ, Recommendations for probiotic use-2011 update. ClinGastroenterol. 2011; 45:S168-71.

18. Chang JY, Antonopoulos DA, Kalra A, Tonelli A, Khalife WT, Schmidt TM, Young VB. Decreased diversity of the fecal microbiome in recurrent clostridium difficileassociated diarrhea. Journal of Infectious Diseases. 2008;197(3):435-438

19. Dr. Mellow, Dr. Schiller, and Dr. Kenneley.American College of Gastroenterology (ACG) 2011 Annual Scientific Meeting and Postgraduate Course; President's Plenary Session II. Presented 2011.

20. Brandt LJ, Borody TJ, Campbell J. Endoscopic fecal microbiota transplantation: "first-line" treatment for severe Clostridium difficile infection? J ClinGastroenterol 2011; 45: 655-657.

21. Dr. Kamepalli, Dr. Louie, and Dr. Moore ID Week 2013. Abstract 89. Presented 2013. 
22. Drossman DA. The functional gastrointestinal disorders and the Rome III process. Gastroenterology 2006; 130: 1377-90.

23. Moayyedi P, Ford AC, Talley NJ, Cremonini F, FoxxOrenstein AE, Brandt LJ, Quigley EM .The efficacy of probiotics in the treatment of irritable bowel syndrome: a systematic review. J Gastroenterol. 2009; 44(1):26-46.

24. P Moayyedi, A C Ford, N J Talley, F Cremonini, A E FoxxOrenstein, L J Brandt, E M M Quigley. The efficacy of probiotics in the treatment of IBS. Gut 2010; 59:325-332.

25. A. P. S. Hungin, C. Mulligan, B. Pot, P. Whorwell, L. Agrèus, P. Fracasso, C. Lionis, J. Mendive, J.-M. Philippart de Foy, G. Rubin, C. Winchester, N. de Wit. Probiotics in the Management of Lower Gastrointestinal Symptoms in Clinical Practice. Aliment PharmacolTher. 2013; 38(8):864-886

26. Guarner F, Khan AG, Garisch J, et al. Probiotics and prebiotics. 2011. Available at: http://www.worldgastro enterology.org/probiotics-prebiotics. html.Accessed 2013

27. Meijer BJ, Dieleman LA. Probiotics in the treatment of human inflammatory bowel diseases: update 2011. J ClinGastroenterol. 2011; 45:139-44.

28. Khan KJ, Ullman TA, Ford AC et al,Antibiotic therapy in inflammatory bowel disease: a systematic review and metaanalysis. Am J Gastroenterol. 2011;106:661-73.

29. SieglindeAngelberger MD, Walter Reinisch MD, AthanasiosMakristathis PhD, Cornelia Lichtenberger et al, Temporal Bacterial Community Dynamics Vary Among Ulcerative Colitis Patients After Fecal Microbiota Transplantation Am J Gastroenterol. 2013;108(10): 1620-1630

30. Naidoo K, Gordon M, Fagbemi AO, Thomas AG, Akobeng AK. Probiotics for maintenance of remission in ulcerative colitis. Cochrane Database Syst Rev. 2011; (12):CD007443.

31. Cain AM, KarpaKD Clinical utility of probiotics in inflammatory bowel disease AlternTher Health Med.2011;17(1):72-79.

32. Floch MH, Walker WA, Madsen K, Sanders ME, Macfarlane GT, Flint HJ, Dieleman LA, Ringel Y, Guandalini S, Kelly CP, Brandt LJ, Recommendations for probiotic use-2011 update J ClinGastroenterol. 2011; 45 (3):168-71

33. Pardi DS, D’Haens G, Shen B, Campbell S, Gionchetti P, Clinical guidelines for the management of pouchitis in Inflamm Bowel Dis. Gut 2009;15(9):1424-31.

34. Humes DJ. Changing epidemiology: does it increase our understanding? J DigDis 2012; 30: 6-11.

35. Tursi A. Antibiotics and probiotics in the treatment of diverticular disease. J ClinGastroenterol. 2011; 45(12): 46-52.

36. Stollman N, Magowan S, Shanahan F, Quigley EM. A randomized controlled study of mesalamine after acute diverticulitis: results of the DIVA trial. J ClinGastroenterol2013; 47: 621-9.

37. Tursi A, G. Brandimarte, W. Elisei, M. Picchio et al, Mesalazine and/or Probiotics in Maintaining Remission of Symptomatic Uncomplicated Diverticular Disease. Aliment PharmacolTher. 2013;38(7):741-751.

38. Bataller R, Brenner DA. Liver fibrosis. J Clin Invest. 2005;115:209-218.

39. Yan AW, Fouts DE, Brandl J, Starkel P, Torralba M, Schott E, Tsukamoto H, Nelson KE, Brenner DA, Schnabl B. Enteric dysbiosis associated with a mouse model of alcoholic liver disease. Hepatology. 2011;53(1):96-105.

40. Murphy EF, Cotter PD, Hogan A, O’Sullivan O, Joyce A, Fouhy F, Clarke SF, Marques TM, O'Toole PW, Stanton $\mathrm{C}$ et al, Divergent metabolic outcomes arising from targeted manipulation of the gut microbiota in diet-induced obesity. Gut.2013; 62(2):220-6.

41. Turnbaugh PJ, Ley RE, Mahowald MA, Magrini V, Mardis ER, Gordon JI. An obesity-associated gut microbiome with increased capacity for energy harvest. Nature. 2006; 444(22):1027-1031.

42. Million M, Lagier JC, Yahav D, Paul M. Gut bacterial microbiota and obesity.ClinMicrobiol Infect. 2013;19(4):305-13.

43. Han J, Lin H.Intestinalmicrobiota and type 2 diabetes: From mechanism insights to therapeutic perspective. World J Gastroenterol. 2014;20(47):17737-17745.

44. Remely M, Aumueller E, Jahn D, Hippe B, Brath H, Haslberger AG.MicrobiotaHYPERLINK "http:// www.ncbi.nlm.nih.gov/pubmed/24533976” and epigenetic regulation of inflammatory mediators in type 2 diabetes and obesityHYPERLINK "http://www.ncbi.nlm.nih.gov/ pubmed/24533976”. Benef Microbes. 2014;5(1):33-43.

45. Naidoo K, Gordon M, Fagbemi AO, Thomas AG, Akobeng AK. Probiotics for maintenance of remission in ulcerative colitis. Cochrane Database Syst Rev.2011; (12): CD007443.

46 Sanders ME, Akkermans LM, Haller D, et al. Safety assessment of probiotics for human use. Gut Microbes 2010;1:164-85.

47. Ilan Youngster, George H. Russell, Christina Pindar, Tomer Ziv-Baran et al, Oral, Capsulized, Frozen Fecal Microbiota Transplantation for Relapsing Clostridium difficile Infection. JAMA. 2014;312(17):1772-1778.

48. Besselink MG, van Santvoort HC, Buskens E, Boermeester MA et al, Probiotic prophylaxis in predicted severe acute pancreatitis: a randomised, double-blind, placebocontrolled trial Dutch Acute Pancreatitis Study Group Lancet. 2008; 23(13):651-9.

49. O’Connell Motherway M, Zomer A, Leahy SCet al,Functional genome analysis of Bifidobacteriumbreve UCC2003 reveals type IVb tight adherence (Tad) pili as an essential and conserved host-colonization factor. ProcNatlAcadSci USA 2011; 108: 11217-22.

50. FAO/WHO Expert Consultation. Health and nutritional properties of probiotics in food including powder milk with live lactic acid bacteria. Report of a joint FAO/WHO expert consultation on evaluation of health and nutritional properties of probiotics in food including powder milk with live lactic acid bacteria, 2001. Cordoba, Argentina. Accessed 2012.

51. Ciorba MA. A gastroenterologist's guide to probiotics. ClinGastroenterolHepatol. 2012; 10 (9):960-8. 